\title{
B-Vitamins, blood pressure and endothelial compliance in healthy adults stratified by MTHFR genotype
}

\author{
M. Rooney, M. Clements, C.F. Hughes, H. McNulty, J.J. Strain and M. Ward \\ Nutrition Innovation Centre for Food and Health (NICHE), Ulster University, Cromore Rd, Coleraine, BT55 1SA, \\ Northern Ireland.
}

Hypertension affects 1 billion people worldwide and is a primary public health concern as the leading cause of premature mortality. A common polymorphism, the C677 T mutation in the gene, MTHFR, encoding the enzyme methylenetetrahydrofolate reductase (MTHFR) affects $12 \%$ of the UK and Irish population and is associated with an increased risk of hypertension ${ }^{(1)}$. The activity of MTHFR, which generates the predominant co-factor form of folate, 5 methyl-tetrahydrofolate, is dependent on flavin adenine dinucleotide (FAD), the co-enzyme form of riboflavin. Individuals homozygous for the MTHFR 677TT genotype have reduced MTHFR enzyme activity resulting in lower 5 methyl-THF concentrations ${ }^{(2)}$, however previous randomised controlled trials at our Centre have shown that blood pressure (BP) is highly responsive to riboflavin supplementation specifically in individuals with the TT genotype ${ }^{(3)}$. The mechanism explaining how this gene-nutrient interaction influences BP is unknown, but it may involve a nitric oxide (NO)-mediated effect on endothelial function ${ }^{(1)}$. The aim of this study was to investigate BP and endothelial function in adults stratified by MTHFR genotype.

Healthy individuals, aged 18-60 years, were recruited from workplaces across Northern Ireland and screened for MTHFR genotype. Participants were invited to an appointment where health and lifestyle information, anthropometry, BP and endothelial function were assessed and a blood sample was taken. Pulse wave velocity (PWV) and augmentation index (AIx), which are indices of endothelial function, were measured with a SphygmoCor (AtCor Medical, Australia). BP, PWV and AIx were compared across the MTHFR genotypes.

\begin{tabular}{|c|c|c|c|c|c|c|c|c|c|}
\hline & \multicolumn{2}{|c|}{ All $(n=108)$} & \multicolumn{2}{|c|}{$\mathrm{CC}(\mathrm{n}=22)$} & \multicolumn{2}{|c|}{ CT $(n=24)$} & \multicolumn{2}{|c|}{ TT $(n=62)$} & \multirow[b]{2}{*}{$\mathrm{P}$} \\
\hline & Mean & SD & Mean & SD & Mean & $\mathrm{SD}$ & Mean & SD & \\
\hline Age (years) & $42 \cdot 32$ & $10 \cdot 49$ & $42 \cdot 05$ & $10 \cdot 25$ & $39 \cdot 67$ & $11 \cdot 39$ & $43 \cdot 45$ & $16 \cdot 19$ & $0 \cdot 384$ \\
\hline Male $(\%)$ & $65(60 \%)$ & & $14(64 \%)$ & & $13(54 \%)$ & & $38(61 \%)$ & & $0 \cdot 512$ \\
\hline SBP mmHg & $133 \cdot 3$ & $14 \cdot 2$ & $128 \cdot 5^{\mathrm{a}}$ & $11 \cdot 0$ & $128 \cdot 0^{\mathrm{a}}$ & $8 \cdot 6$ & $137 \cdot 1^{\mathrm{b}}$ & $15 \cdot 78$ & $<0.001$ \\
\hline DBP $\mathrm{mmHg}$ & $81 \cdot 0$ & $10 \cdot 7$ & $77 \cdot 7$ & $7 \cdot 7$ & $80 \cdot 4$ & 9.4 & $82 \cdot 5$ & 9.4 & $0 \cdot 112$ \\
\hline AIx $(\%)$ & $20 \cdot 19$ & $14 \cdot 31$ & $14 \cdot 36$ & $14 \cdot 00$ & $21 \cdot 21$ & $16 \cdot 90$ & $21 \cdot 85$ & $12 \cdot 99$ & $0 \cdot 118$ \\
\hline PWV (m/s) & $7 \cdot 13$ & 1.44 & $6 \cdot 42^{\mathrm{a}}$ & $1 \cdot 15$ & $7 \cdot 33^{\mathrm{ab}}$ & 1.66 & $7 \cdot 30^{\mathrm{b}}$ & $1 \cdot 38$ & $0 \cdot 035$ \\
\hline
\end{tabular}

Differences between groups were assessed by ANOVA; values within a row with different superscript letters are significantly different, by Tuckey post-hoc test Abbreviations: AIx, augmentation index; DBP, diastolic blood pressure; PWV, pulse wave velocity; SBP. Systolic BP.

Preliminary results in a subset of the study cohort, summarised in the table above, show that systolic BP is markedly higher in participants with the TT genotype, compared to CC and CT genotypes, with a similar, albeit non-significant, trend for diastolic BP. Pulse wave velocity was significantly higher in individuals with the TT compared to CC genotype, indicating poorer endothelial compliance in this genetically at-risk group.

In conclusion, this study has shown for the first time that individuals with the MTHFR TT genotype have poorer endothelial function compared to their age-matched CC genotype counterparts. Further research investigating the effect of riboflavin (and folate) status on measures of vascular function is required, in order to further our understanding of the mechanism explaining this novel gene-nutrient interaction in BP.

\section{Acknowledgements}

Northern Ireland Clinical Research Facility (NICRF), City Hospital, Belfast.

1. McNulty H, Strain JJS, Hughes CF et al. (2017) Mol Aspects Med 53, 2-9.

2. Antoniades C, Shirodaria C, Leeson P et al. (2009) Circulation 119 (18), 2507-15.

3. Horigan G, McNulty H, Ward M, et al. (2010) J Hypertens 28 (3), 478-86. 